\title{
PLANNED LEAD TIMES IN MRP, COST CONSIDERATIONS AND CUSTOMER SERVICE
}

\section{Ton G. de Kok, Eindhoven University of Technology}

In this presentation we discuss the problem of the determination of the planned lead time parameters in MRP systems. MRP systems are widely used in practice in job shop and mass assembly production systems. The MPP logic translates customer demand into time-phased demand for sub-assemblies and components. The time-phasing is based on the planned lead times of purchasing and sub-assembly. In most situations the planned lead times are estimated based on average and standard deviations of the actual lead time parameters. E.g. the planned lead time is set in such a way that the probability that the actual lead time is shorter than the planned lead time equals $90 \%$. We present an overview of methods to arrive at the planned lead times.

In addition we present a model in which both Work-In-Progress costs and customer service criteria are taken into account to find an optimal set of planned lead times for a serial production system with stochastic lead times. Using the model we can find rules of thumb for setting planned lead times in most practical situations.

The analysis of the model turns out to allow for a full decomposition of the model. I.e. for an N-stage serial production system we have to recursively solve stage by stage an equation, which is similar to that arising in the classical Newsboy problem.

Since the equations turn out to be rather complicated expressions, we have developed an approximation scheme, which gives very accurate results in negligible time. An important problem is the determination of the actual lead time distribution. We will discuss practically useful results from queuing theory to arrive at these lead time distributions. 